le portiQue Le Portique

Revue de philosophie et de sciences humaines

$10 \mid 2002$

Les paradis artificiels

\title{
Petite lecture des « Trois essais sur la théorie sexuelle »
}

\section{Raphaël Herr}

\section{(2) OpenEdition}

1 Journals

Édition électronique

URL : http://journals.openedition.org/leportique/160

DOI : 10.4000/leportique.160

ISSN : $1777-5280$

Éditeur

Association "Les Amis du Portique"

Édition imprimée

Date de publication : 1 septembre 2002

ISSN : 1283-8594

Référence électronique

Raphaël Herr, «Petite lecture des «Trois essais sur la théorie sexuelle » », Le Portique [En ligne], 10 | 2002, mis en ligne le 06 juin 2005, consulté le 08 avril 2021. URL : http://journals.openedition.org/ leportique/160 ; DOI : https://doi.org/10.4000/leportique.160

Ce document a été généré automatiquement le 8 avril 2021.

Tous droits réservés 


\title{
Petite lecture des « Trois essais sur la théorie sexuelle»
}

\author{
Raphaël Herr
}

1 Ce texte est la réécriture d'une intervention faite à l'occasion d'une journée d'étude sur la question de l'Oralité, organisée par le Collège des psychologues du Centre Hospitalier de Jury-les-Metz, au mois de novembre 2002. La proximité de cette question avec celle de drogues et de paradis artificiels qui ne seraient pas de purs semblants avait de quoi retenir notre attention.

2 Il s'agissait pour nous, chargé d'introduire les travaux, de rappeler à un large public le vocabulaire freudien minimal, qui permet de penser la mécanique pulsionnelle dite orale. Davantage, puisque nous avions choisi, ce jour-là, un thème dont l'ambiguïté témoigne de la rencontre, voire de l'enchevêtrement du discours médical et du discours analytique, nous nous devions de fournir à l'assemblée les moyens conceptuels nécessaires à leur distinction.

3 L'ouvrage de Freud, publié en 1905, sous le titre original Drei Abhandlungen zur Sexualtheorie ${ }^{1}$, présente, merveilleusement rassemblée dans le chapitre consacré au suçotement, une série d'énoncés qui se prêtent parfaitement à une initiation publique à la grammaire de la psychanalyse.

4 Dans le deuxième essai, «Les manifestations de la sexualité infantile », Freud fait du suçotement l'image même de la poussée orale : il s'agit... " d'une répétition rythmique, avec la bouche, d'un contact de succion, dont la finalité alimentaire est exclue " ${ }^{2}$.

5 À l'heure où, dans le langage courant, il n'est plus question que de troubles des conduites, notamment alimentaires, du fait d'influences comportementalistes massives, nous mesurons immédiatement qu'il s'agit là d'autre chose. La clinique freudienne n'est pas du côté des comportements finalisés, mais de la répétition, quasi hallucinatoire, d'un contact qui a marqué le champ de la satisfaction, pour un sujet.

6 Plus loin : « il n'est pas rare que la friction de certaines parties sensibles du corps, de la poitrine, des organes génitaux externes, se combine avec la succion voluptueuse. Beaucoup d'enfants passent par cette voie, du suçotement à la masturbation... » En psychanalyse, le corps est 
bien différent de celui qui occupe l'anatomiste. Il se situe au bord, mieux, à l'envers de l'organisme, dans un autre lieu, invisible et inouï, où il s'agit de l'inscription des traces laissées par des expériences de jouissance.

7 La jouissance est un concept pratique, qui exprime sous une forme lapidaire, la complexité et la profonde ambivalence de ces expériences de plaisir: le plus souvent, Freud parlait du couple, inséparable, formé par le plaisir et le déplaisir.

8 L'autre lieu, quant à lui, est parfois appelé l'autre scène : cette image symbolise l'idée d'un espace qui n'apparait pas au regard: il faut se donner un peu de mal pour l'entendre. Prétendre écouter l'autre est aujourd'hui une profession de foi vulgarisée et édulcorée: le concept d'inconscient, ou d'insu, appelle une oreille affûtée à la singularité de la chose.

9 Un peu avant, dans le premier Essai, Freud semble se livrer à un curieux recensement des «aberrations sexuelles ». Non pas comme un hygiéniste qui serait guidé par une morale rigoureuse, mais afin de conclure à la nécessité - pour pouvoir penser effectivement- de relâcher les liens entre la pulsion et l'objet. «Il est probable - nous dit-il - que la pulsion sexuelle est d'abord indépendante de son objet ».

11 Reste à savoir si Freud a, un jour, prétendu qu'elle finissait par le trouver... L'étymologie du mot «aberration » nous indique plutôt un éloignement, une errance, dont rien ne nous garantit la fin heureuse et normative !

À ce stade, il est bon de disposer d'une définition du concept de pulsion :

13 il s'agit d'une poussée, interne, c'est-à-dire interne à l'appareil psychique, dont on ne connaît que la manifestation. Elle agit sur le mode d'une force constante - devant laquelle rien ne sert de fuir : on ne se dérobe pas devant quelque chose qui vous attend " chez vous » - dont le but consiste à se satisfaire. Par exemple, le but de la pulsion orale est l'incorporation.

14 Continuons notre lecture :

«il est clair...que l'acte de l'enfant qui suçote est déterminé par la recherche d'un plaisir déjà vécu....et désormais remémoré ».

15 La réalité dont il est ici question est une réalité psychique, où il s'agit d'empreintes, de traces, d'idées. Le psychisme est un monde tissé de représentations, gérant des excitations, qui viennent toujours de l'intérieur. Pour comprendre un peu ce qu'est l'univers névrotique, nul besoin de faire appel à un traumatisme réel.

16 Freud caressait quelques dix ans auparavant, l'idée d'une séduction sexuelle, par le père, venant expliquer les symptômes hystériques développés par ses patients. Puis il a renoncé à cette théorie, en fondant véritablement la psychanalyse grâce au concept de fantasme.

17 Le fantasme est un réel en lui-même qui s'accommode parfaitement d'une réalité traumatique ou non!

18 «La première et la plus vitale des activités de l'enfant, la tétée du sein maternel... a déjà du le familiariser avec ce plaisir... les lèvres de l'enfant ont tenu le rôle d'une zone érogène ", soit l'inscription d'une ouverture produite sur le corps par la rencontre avec l'énigme de la jouissance d'un autre, par exemple de la mère, personnage indispensable à la scène; "et la stimulation réalisée par l'afflux du lait chaud fut la cause de la sensation de plaisir ». 
«Au début, la satisfaction de la zone érogène était sans doute associée à la satisfaction du besoin alimentaire... " Il s'agit du concept d'étayage, qui désigne l'appui originel des pulsions sexuelles sur les pulsions de conservation, et au-delà, l'articulation même du somatique avec le psychique. Ou encore, la psychanalyse est d'abord clinique: une clinique de la singularité de cette articulation. Et même lorsqu'il lui est nécessaire de fonctionner comme un discours, pour transmettre le savoir qui est le sien, il ne s'agit ni d'une philosophie, ni, un bien entendu, d'une pédagogie, fusse-t-elle de santé.

"...puis le besoin de répétition se sépare du besoin de nutrition... » Il y a un passage nécessaire par la séparation. La perte d'un lien est constitutive du sujet, c'est-à-dire, d'un individu humanisé par une privation. Ce qui fait que l'enfant investit son pouce, notamment : " pour la succion... l'enfant ne se sert pas d'un objet étranger, mais de préférence d'un endroit de son propre épiderme... »

21 L'humour est manifeste, lorsque Freud envisage la manière dont les reliquats de la première satisfaction déterminent les identités sexuées :

« si... la signification érogène subsiste (à l'âge adulte) ces enfants deviendront de friands amateurs de baisers... ou, si ce sont des hommes, auront un sérieux motif pour boire et pour fumer».

22 L'on admirera ici la geste freudienne en matière de répartition de la jouissance des sexes : il est évidemment scandaleux, au regard de l'égalité des droits, d'entendre que les hommes qui aimeraient les baisers... ou les femmes qui utiliseraient ce motif pour boire seraient dans une profonde erreur quant à leur identité !

Enfin, ces dernières lignes nous mènent sur le vaste territoire de la psychopathologie :

«mais qu'intervienne le refoulement, et ils ressentiront du dégoût pour la nourriture et produiront des vomissements hystériques. En vertu de la double signification de la zone labiale, le refoulement se portera sur la pulsion alimentaire. Un grand nombre de mes patientes affectées de troubles de l'alimentation, de globus hystérique, de sensations d'étranglement et de vomissements ont été d'énergiques suçoteuses durant leur enfance. »

Le but de la pulsion orale consiste à incorporer, mais il convient de préciser, pour terminer, que le destin «normal » d'une pulsion lui promet de rencontrer la castration. Elle parviendra ainsi à emprunter le chemin de la sublimation, et le Sujet saura dès lors, à peu près, distinguer les objets internes de ceux qu'il convient de rencontrer dans la vie courante.

Le domaine clinique de la psychanalyse peut être affiné : elle s'intéresse à tout ce qui fait que chez un être humain, la séparation n'est jamais complètement réussie, ou achevée.

26 C'est dire que le risque d'un retour sauvage de la satisfaction hallucinatoire demeure chez la plupart d'entre nous. Si l'abondance des produits favorise une culture qui met l'objet aux commandes, vouloir faire coïncider l'autre avec une représentation préalable est une folie bien plus ordinaire, qui mérite également toute notre vigilance. 


\section{NOTES}

1.. S. FREUD, Trois essais sur la théorie sexuelle, traduction Philippe Koeppel, Paris, Folio Essais. 2.. Ibid., p. 102-106.

\section{RÉSUMÉS}

La question des drogues et des paradis artificiels est l'occasion d'un retour à un texte original de la découverte freudienne et à sa relecture.

Taking some interest about drugs and "artificial paradises" was the opportunity to go back to one of the first works of Freud's research and to it's reading. 